\title{
On the Lie integrability theorem for the Chaplygin ball
}

\author{
A V Tsiganov \\ St.Petersburg State University, St.Petersburg, Russia \\ e-mail: andrey.tsiganov@gmail.com
}

\begin{abstract}
The necessary number of commuting vector fields for the Chaplygin ball in the absolute space is constructed. We propose to get these vector fields in framework of the Poisson geometry similar to the Hamiltonian mechanics.
\end{abstract}

\section{Introduction}

As in [5] we consider the rolling of a dynamically balanced ball on horizontal absolutely rough table without slipping or sliding. 'Dynamically balanced' means that the geometric center coincides with the center of mass, but the mass distribution is not assumed to be homogeneous. Because of the roughness of the table this ball cannot slip, but it can turn about the vertical axis without violating the constraints.

Let $M=\left(M_{1}, M_{2}, M_{3}\right)$ be the angular momentum of the ball with respect to the contact point and $\omega=\left(\omega_{1}, \omega_{2}, \omega_{3}\right)$ be the angular velocity vector of the rolling ball. Its mass, inertia tensor and radius will be denoted by $m, \mathbf{I}=\operatorname{diag}\left(I_{1}, I_{2}, I_{3}\right)$ and $b$ respectively. Here and below all the vectors are expressed in the so-called body frame, which is firmly attached to the ball, and its axes coincide with the principal inertia axes of the ball.

Equations of motion

$$
\dot{\alpha}=\alpha \times \omega, \quad \dot{\beta}=\beta \times \omega, \quad \dot{\gamma}=\gamma \times \omega, \quad \dot{M}=M \times \omega,
$$

have the same form as the Euler-Poisson equations in rigid body dynamics [5, 4, 7]. In fact the principal difference between holonomic and nonholonomic systems is chidden within the relation of the angular velocity with the angular momentum

$$
\omega=\mathbf{A}_{\gamma} M
$$

where in the Chaplygin case

$$
\mathbf{A}_{\gamma}=\mathbf{A}+\frac{d \mathbf{A} \gamma \otimes \gamma \mathbf{A}}{g^{2}(\gamma)}, \quad \mathbf{A}=\left(\begin{array}{ccc}
a_{1} & 0 & 0 \\
0 & a_{2} & 0 \\
0 & 0 & a_{3}
\end{array}\right), \quad a_{k}=\left(I_{k}+d\right)^{-1}
$$

and

$$
g(\gamma)=\sqrt{1-d(\gamma, \mathbf{A} \gamma)}, \quad d=m b^{2} .
$$

Here and below we always assume that unit vector $\gamma$ is perpendicular to the plane.

Remark 1 At $d=0$ one gets standard Euler-Poisson equations for the Euler-Poinsot top, which is a well-studied holonomic Hamiltonian dynamical system.

According to the kinematic interpretation, the first three equations in (1.1) describe the evolution of a fixed in the space ortonormal inertial frame rotating with the angular velocity $\omega$. Basis unit vectors

$$
\alpha=\left(\alpha_{1}, \alpha_{2}, \alpha_{3}\right), \quad \beta=\left(\beta_{1}, \beta_{2}, \beta_{3}\right), \quad \gamma=\left(\gamma_{1}, \gamma_{2}, \gamma_{3}\right)
$$


of the inertial frame expressed in the rotating reference frame are columns of the real orthogonal rotation matrix

$$
\mathbf{R}=\left(\begin{array}{lll}
\alpha_{1} & \beta_{1} & \gamma_{1} \\
\alpha_{2} & \beta_{2} & \gamma_{2} \\
\alpha_{3} & \beta_{3} & \gamma_{3}
\end{array}\right) \in S O(3)
$$

Consequently vector field $X$ defined by (1.1) has six geometric or kinematic integrals of motion

$$
\begin{array}{lll}
C_{1}=(\alpha, \alpha), & C_{2}=(\beta, \beta), & C_{3}=(\gamma, \gamma), \\
C_{4}=(\alpha, \beta), & C_{5}=(\alpha, \gamma), & C_{6}=(\beta, \gamma),
\end{array}
$$

which are equal to one $\left(C_{1}, \ldots, C_{3}\right)$ or to zero $\left(C_{4}, \ldots, C_{6}\right)$.

Because ball is a free rigid body not subject to any external force, spatial components of the angular momentum

$$
J_{1}=(\alpha, M), \quad J_{2}=(\beta, M), \quad J_{3}=(\gamma, M)
$$

are three integrals of motion and replacing one of them by the sum of their squares yields a second quadratic in momenta integral of motion

$$
M^{2}=M_{1}^{2}+M_{2}^{2}+M_{3}^{2}=J_{1}^{2}+J_{2}^{2}+J_{3}^{2}
$$

as well as the kinetic energy

$$
H=\frac{1}{2}(M, \omega)
$$

According to [5] vector field $X$ has an invariant volume form

$$
\Omega=g^{-1}(\gamma) \mathrm{d} \alpha \wedge \mathrm{d} \beta \wedge \mathrm{d} \gamma \wedge \mathrm{d} M
$$

Thus, according to the Euler-Jacobi theorem it is possible to integrate the system (1.1) by quadratures.

The main aim of this paper is to prove the integrability of the vector field $X$ (1.1) by means of the Lie integrability theorem.

\subsection{The Euler-Jacobi and Lie integrability theorems}

Let us remind of some facts about various integrability theorems following the recent work [9].

According to Euler and Jacobi, vector field $X$ defined by

$$
\dot{x}_{i}=X_{i}\left(x_{1}, \ldots, x_{n}\right)
$$

is integrable by quadratures if there are $n-2$ functionally independent first integrals $f_{1}, \ldots, f_{n-2}$ and an invariant volume form

$$
\Omega=\mu \mathrm{d} x_{1} \wedge \mathrm{d} x_{2} \wedge \cdots \wedge \mathrm{d} x_{n}
$$

In the classical mechanics this assertion is often referred to as the Euler-Jacobi theorem of the last multiplier. In this case the compact connected component of the level set of integrals of motion without singular points is a smooth manifold diffeomorphic to the two-dimensional torus, see [1].

A classical Lie theorem [11] says that the vector field $X$ (1.10) is integrable by quadratures if there are $n$ independent vector fields

$$
u_{1}=X, u_{2}, \ldots, u_{n}
$$

which generate a solvable Lie algebra with respect to the commutation operation [., .], modern discussion may be found in [9, 14]. 
The Jacobi last multiplier $\mu$ (1.11) provides an incestuous interrelation between symmetries, first integrals and integrating factors for well-endowed systems, see Lie [11], page 343-347 and Bianchi [2] pages 456-464.

For instance, if we know all $n$ generators (1.12) of the symmetry algebra

$$
u_{i}=\sum_{j=1}^{n} a_{i j} \partial_{x_{j}}, \quad i=1, \ldots, n,
$$

the Jacobi last multiplier (1.11) is also given by

$$
\mu=g^{-1}, \quad g=\operatorname{det}\left[\begin{array}{cccc}
a_{11} & a_{12} & \cdots & a_{1 n} \\
\vdots & & & \vdots \\
a_{n 1} & a_{n 2} & \cdots & a_{n n}
\end{array}\right]
$$

in case of $g \neq 0$. So, in the classical Lie integrability theorem we usually have $n+1$ tensor invariants.

In generic cases the vector field $X(1.10)$ is integrable by quadratures if there are $k$ functional independent integrals of motion

$$
f_{1}, \ldots, f_{k}
$$

and $n-k$ linearly independent vector fields

$$
u_{1}=X, u_{2}, \ldots, u_{n-k}
$$

which generate a solvable Lie algebra, and the Lie derivatives along any of the fields $u_{i}$ from any of the integrals $f_{j}$ are equal to zero

$$
\mathcal{L}_{u_{i}} f_{j}=0 .
$$

The above statement follows immediately from the Lie theorem applied to the restriction of the dynamical system (1.10) on the integral manifold

$$
\mathcal{N}_{c}=\left\{x \in \mathcal{M}, \quad f_{1}=c_{1}, \ldots, f_{k}=c_{k}\right\} .
$$

In fact, the Lie integrability theorem obviously implies the well-known Liouville theorem on the integrability of the Hamiltonian vector field with a complete set of independent integrals of motion in involution. In this simplest case $n=2 k$, vector field $X$ (1.10) is the Hamiltonian vector field

$$
u_{1}=X=P \mathrm{~d} H, \quad H=f_{1},
$$

and $k$ functional independent integrals of motion $f_{j}$ (1.14) are in involution

$$
\left\{f_{i}, f_{j}\right\}=\sum_{l m=1}^{n} P_{l m} \frac{\partial f_{i}}{\partial x_{l}} \frac{\partial f_{j}}{\partial x_{m}}=0, \quad i, j=1, \ldots, k,
$$

with respect to the Poisson brackets defined by the Poisson bivector $P$. So $k$ vector fields

$$
u_{i}=P \mathrm{~d} f_{i}
$$

are linearly independent at each point, they commute pairwise and relations (1.16) hold true. See 1, 10, 13] for a more comprehensive discussion on integrability theorems and related issues.

Two important consequences of the Jacobi identity for $P$ are that the generalized distribution on $\mathcal{M}$, defined by the Hamiltonian vector $X=P d H$, is integrable, and that the Hamiltonian vector fields (1.18) which are associated with Poisson commuting functions (1.17) are commuting vector fields. 


\section{Linear Poisson bivectors for the Chaplygin ball}

In the holonomic case at $d=0$ the vector field $X$ (1.1) is a Hamiltonian vector field

$$
X=P \mathrm{~d} H
$$

with respect to the Hamiltonian (1.8) and to the canonical Poisson bivector

$$
P=\left(\begin{array}{cccc}
0 & 0 & 0 & \boldsymbol{A} \\
0 & 0 & 0 & \boldsymbol{B} \\
0 & 0 & 0 & \boldsymbol{\Gamma} \\
\boldsymbol{A} & \boldsymbol{B} & \boldsymbol{\Gamma} & \boldsymbol{M}
\end{array}\right)
$$

Here we use a convention that any vector $z$ is identified with the $3 \times 3$ skew-symmetric matrix $Z$

$$
z=\left(\begin{array}{c}
z_{1} \\
z_{2} \\
z_{3}
\end{array}\right) \rightarrow \boldsymbol{Z}=\left(\begin{array}{ccc}
0 & z_{3} & -z_{2} \\
-z_{3} & 0 & z_{1} \\
z_{2} & -z_{1} & 0
\end{array}\right)
$$

Remark 2 At $d=0$ it is easy to see that the following change of variables

$$
\gamma \rightarrow \gamma=\frac{\gamma \times M}{|\gamma \times M|}
$$

preserves the equations of motion (1.1) and reduces the linear in momenta Poisson bivector $P$ (2.1) to a rational Poisson bivector. In order to avoid such freedom below we will consider only linear in momenta Poisson bivectors.

Integrals of motion (1.6) are the Casimir functions of $P$ (2.1)

$$
P \mathrm{~d} C_{k}=0,
$$

which give rise to the trivial vector fields. Spatial components of the angular momentum (1.7) generate $s o(3)$ algebra

$$
\left\{J_{i}, J_{j}\right\}=\varepsilon_{i j k} J_{k}
$$

where $\varepsilon_{i j k}$ is a totally skew-symmetric tensor. The two remaining integrals of motion $H$ and $M^{2}=(M, M)$ are in involution with all the other integrals .

Thus, in the holonomic Euler case at $d=0$ we have nine functionally independent integrals of motion

$$
C_{1}, \ldots, C_{6}, \quad f_{1}=H, \quad f_{2}=M^{2}, \quad f_{3}=(\gamma, M),
$$

and three commuting to each other vector fields

$$
u_{1}=P \mathrm{~d} f_{1}, \quad u_{2}=P \mathrm{~d} f_{2}, \quad u_{3}=P \mathrm{~d} f_{3},
$$

so that

$$
\mathcal{L}_{u_{i}} f_{j}=0, \quad \forall i, j .
$$

It allows us to say that vector field $X$ can be integrable by quadratures according to the Lie integrability theorem.

\subsection{Nonholonomic case}

As in [5] let us start with the projection of the initial vector field $X$ (1.1)

$$
\hat{X}: \quad \dot{\gamma}=\gamma \times \omega, \quad \dot{M}=M \times \omega
$$


on the six-dimensional phase space $\hat{\mathcal{M}}$ with coordinates $\gamma$ and $M$. According to 4 the vector field $\hat{X}$ is a conformally Hamiltonian vector field

$$
\hat{X}=g^{-1} \hat{P}_{g} \mathrm{~d} H
$$

with respect to the Poisson bivector

$$
\hat{P}_{g}=g\left(\begin{array}{cc}
0 & \boldsymbol{\Gamma} \\
\Gamma & \mathbf{M}
\end{array}\right)-d g^{-1}(M, \mathbf{A} \gamma)\left(\begin{array}{cc}
0 & 0 \\
0 & \boldsymbol{\Gamma}
\end{array}\right) .
$$

Integrals of motion $(\gamma, M)$ and $(\gamma, \gamma)$ are the Casimir functions of $\hat{P}_{g}$ and two remaining integrals of motion $H$ and $M^{2}$ are in involution with respect to the corresponding Poisson brackets. It allows us to get two commuting Hamiltonian vector fields

$$
v_{1}=\hat{P}_{g} \mathrm{~d} H, \quad v_{2}=\hat{P}_{g} \mathrm{~d} M^{2},
$$

and to apply the Lie integrability theorem after changing the time $\mathrm{d} t \rightarrow g^{-1} \mathrm{~d} t$ as proposed by Chaplygin [5].

It is easy to represent initial vector field $X$ (1.1) in the same form

$$
X=g^{-1} \widetilde{P}_{g} \mathrm{~d} H
$$

using an almost Poisson bivector

$$
\widetilde{P}_{g}=g\left(\begin{array}{cccc}
0 & 0 & 0 & \boldsymbol{A} \\
0 & 0 & 0 & \boldsymbol{B} \\
0 & 0 & 0 & \boldsymbol{\Gamma} \\
\boldsymbol{A} & \boldsymbol{B} & \boldsymbol{\Gamma} & \boldsymbol{M}
\end{array}\right)-d g^{-1}(M, \mathbf{A} \gamma)\left(\begin{array}{cccc}
0 & 0 & 0 & 0 \\
0 & 0 & 0 & 0 \\
0 & 0 & 0 & 0 \\
0 & 0 & 0 & \boldsymbol{\Gamma}
\end{array}\right) .
$$

We have to underline that initial vector field field $X$ ceases to be a conformally Hamiltonian vector field on $\mathcal{M}$, $\operatorname{dim} \mathcal{M}=12$ as bivector $\widetilde{P}_{g}$ does not satisfy to the Jacobi identity and, thus, the field $\widetilde{P}_{g} \mathrm{~d} H$ is non-Hamiltonian.

Below we prove that there are several reductions of this almost-Poisson bivector to Poisson bivectors. Let us describe one partial example.

Proposition 1 Bivector

$$
P_{g}=\widetilde{P}_{g}+\left(\begin{array}{cccc}
0 & 0 & 0 & \boldsymbol{A}_{g} \\
0 & 0 & 0 & \boldsymbol{B}_{g} \\
0 & 0 & 0 & 0 \\
-\boldsymbol{A}_{g}^{\top} & -\boldsymbol{B}_{g}^{\top} & 0 & 0
\end{array}\right)
$$

where

$$
\left(\boldsymbol{A}_{g}\right)_{i j}=(\alpha \times \gamma)_{i} \sigma_{j}, \quad\left(\boldsymbol{B}_{g}\right)_{i j}=(\beta \times \gamma)_{i} \sigma_{j}
$$

and

$$
\sigma_{1}=\frac{\gamma_{1}}{\gamma_{1}^{2}+\gamma_{2}^{2}}\left(1+g-\gamma_{3} \sigma_{3}\right), \quad \sigma_{2}=\frac{\gamma_{2}}{\gamma_{1}} \sigma_{1}, \quad \sigma_{3}=\frac{d \gamma_{3}\left(a_{1} \gamma_{1}^{2}+a_{2} \gamma_{2}^{2}\right)}{d\left(a_{1} \gamma_{1}^{2}+a_{2} \gamma_{2}^{2}\right)-\gamma_{1}^{2}-\gamma_{2}^{2}} .
$$

is a Poisson bivector. The Casimir functions of this Poisson bivector $P_{g}$, rank $P_{g}=6$,

$$
P_{g} \mathrm{~d} C_{k}=0, \quad k=1, \ldots, 6,
$$

coincide with geometric integrals $C_{1}, \ldots, C_{6}$ (1.6).

The proof is a straightforward verification.

Unit vectors $\alpha, \beta$ and $\gamma$ form an inertial orthonormal frame, so we can rewrite entries of $\boldsymbol{A}_{g}$ and $\boldsymbol{B}_{g}$ in the following way

$$
\left(\boldsymbol{A}_{g}\right)_{i j}=-\beta_{i} \sigma_{j}, \quad\left(\boldsymbol{B}_{g}\right)_{i j}=\alpha_{i} \sigma_{j} .
$$


Remark 3 Bivector $P_{g}$ is a Turiel type deformation of the canonical bivector $P$ [2.1 and, therefore, there is a family of Poisson maps which reduce $P_{g}$ to canonical form [17, 18. We present only one partial mapping $M \rightarrow L$, which reduces $P_{g}$ to $P$ (2.1)

$$
\begin{aligned}
& L_{1}=g^{-1}\left(M_{1}+\frac{\left(g-\gamma_{3} \sigma_{3}-1\right)(\gamma, M)}{\gamma_{1}^{2}+\gamma_{2}^{2}} \gamma_{1}\right), \quad L_{3}=g^{-1}\left(M_{3}+\sigma_{3}(\gamma, M)\right), \\
& L_{2}=g^{-1}\left(M_{2}+\frac{\left(g-\gamma_{3} \sigma_{3}-1\right)(\gamma, M)}{\gamma_{1}^{2}+\gamma_{2}^{2}} \gamma_{2}\right),
\end{aligned}
$$

so that

$$
(\gamma, M)=(\gamma, L)
$$

According to [17, 18, there are other Poisson maps expressed via elliptic integrals, which do not have singularity at $\gamma_{1,2}=0$.

Using the Poisson bivector $P_{g}(2.5)$ we can easily prove that the nine functionally independent integrals of motion

$$
C_{1}, \ldots, C_{6}, \quad f_{1}=H, \quad f_{2}=M^{2}, \quad f_{3}=(\gamma, M)
$$

are in involution with respect to the corresponding Poisson bracket $\{., .\}_{g}$. Spatial components $J_{k}$ (1.7) of momenta have the following brackets

$$
\left\{J_{2}, J_{3}\right\}_{g}=J_{1}, \quad\left\{J_{1}, J_{3}\right\}_{g}=-J_{2},
$$

whereas bracket $\left\{J_{1}, J_{2}\right\}_{g}$ is not an integral of motion in contrast with the holonomic case (2.2).

Three independent integrals of motion $f_{k}$ in involution give rise to linear independent commuting vector fields

$$
u_{2}=P_{g} \mathrm{~d} H, \quad u_{3}=P_{g} \mathrm{~d} M^{2}, \quad u_{4}=P_{g} \mathrm{~d}(\gamma, M), \quad\left[u_{i}, u_{j}\right]=0 .
$$

The initial vector field $X$ (1.1) is a linear combination

$$
X=g^{-1}\left(u_{2}-s_{1} u_{4}\right)
$$

of these Hamiltonian vector fields, where

$$
s_{1}=\left(a_{1} \gamma_{1} M_{1}+a_{2} \gamma_{2} M_{2}\right)\left(\frac{1-d a_{3} \gamma_{3}^{2}}{g\left(\gamma_{1}^{2}+\gamma_{2}^{2}\right)}-\frac{1}{d\left(a_{1} \gamma_{1}^{2}+a_{2} \gamma_{2}^{2}\right)-\gamma_{1}^{2}-\gamma_{2}^{2}}\right)+\frac{\left(a_{1} \gamma_{1}^{2}+a_{2} \gamma_{2}^{2}\right) d a_{3} \gamma_{3} M_{3}}{g\left(\gamma_{1}^{2}+\gamma_{2}^{2}\right)} .
$$

Similar decompositions (2.9) of the original vector field on the Hamiltonian vector fields for other nonholonomic systems are discussed in [3, 19, 20].

In contrast with the holonomic case in $d \neq 0$ vector field $X$ has only one symmetry field

$$
u_{4}=P_{g} \mathrm{~d}(\gamma, M), \quad\left[X, u_{4}\right]=0,
$$

because

$$
\left[X, u_{2}\right] \neq 0, \quad\left[X, u_{3}\right] \neq 0 .
$$

In order to get the missing symmetry vector field we can apply the Chaplygin change of time

$$
\mathrm{d} t \rightarrow g^{-1} \mathrm{~d} t
$$

and define three independent vector fields

$$
v_{1}=g X=u_{2}-s_{1} u_{4}, \quad v_{2}=u_{3}-s_{2} u_{4}, \quad v_{3}=u_{4} .
$$

If coefficient $s_{2}$ satisfies the relation

$$
\mathcal{L}_{u_{2}} s_{2}=\mathcal{L}_{u_{3}} s_{1}, \quad \mathcal{L}_{u_{4}} s_{2}=0
$$


these vector fields $v_{1}, v_{2}$ and $v_{3}(2.10$ generate an Abelian algebra with respect to the standard commutator $[.,$.$] .$

After integration of (2.11) one gets an explicit expression for this coefficient

$$
\begin{aligned}
s_{2} & =2\left(\gamma_{1} M_{1}+\gamma_{2} M_{2}\right)\left(\frac{d\left(a_{1} \gamma_{1}^{2}+a_{2} \gamma_{2}^{2}\right)-1}{d\left(a_{1} \gamma_{1}^{2}+a_{2} \gamma_{2}^{2}\right)-\gamma_{1}^{2}-\gamma_{2}^{2}}+\frac{1-d a_{3} \gamma_{3}^{2}}{g\left(\gamma_{1}^{2}+\gamma_{2}^{2}\right)}\right) \\
& +\frac{2 d \gamma_{1} \gamma_{2}\left(a_{1}-a_{2}\right)\left(\gamma_{2} M_{1}-\gamma_{1} M_{2}\right)}{g\left(\gamma_{1}^{2}+\gamma_{2}^{2}\right)}+2 d \gamma_{3} M_{3}\left(\frac{a_{1} \gamma_{1}^{2}+a_{2} \gamma_{2}^{2}}{d\left(a_{1} \gamma_{1}^{2}+a_{2} \gamma_{2}^{2}\right)-\gamma_{1}^{2}-\gamma_{2}^{2}}+\frac{a_{3}}{g}\right) .
\end{aligned}
$$

Thus, we constructed a necessary set of commuting vector fields. It is easy to prove that

$$
\mathcal{L}_{v_{i}} f_{j}=0, \quad i, j=1,2,3,
$$

and

$$
\mathcal{L}_{v_{1}} J_{1,2}=\mathcal{L}_{v_{2}} J_{1,2}=0 .
$$

Here $J_{1,2}$ the spatial components of momenta.

Proposition 2 For the Chaplygin ball vector field $X$ (1.1) at 12-th dimensional phase space $\mathcal{M}$ we can adduce nine functionally independent integrals of motion

$$
C_{1}, \ldots, C_{6}, \quad f_{1}=H, \quad f_{2}=M^{2}, \quad f_{3}=(\gamma, M)
$$

and three linearly independent vector fields commuting to each other's

$$
v_{1}=g X=P_{g} \mathrm{~d} f_{1}-s_{1} P_{g} \mathrm{~d} f_{3}, \quad v_{2}=P_{g} \mathrm{~d} f_{2}-s_{2} P_{g} \mathrm{~d} f_{3}, \quad v_{3}=P_{g} \mathrm{~d} f_{3},
$$

including original field after the time change.

Because the Lie derivatives along any of the fields $v_{i}$ from any of the integrals $f_{j}$ are equal to zero, it is enough for $X$ to be integrable by quadratures according to the Lie integrability theorem.

It is a formal proof of integrability, the constructive procedure of integrability by quadratures has been proposed by Chaplygin [5], see also [7] and references within.

Remark 4 By definition the Hamiltonian vector fields $u_{1}, u_{2}$ and $u_{3}$ (2.8) are Poisson vector fields for the Poisson bivector $P_{g}$

$$
\mathcal{L}_{u_{i}} P_{g}=0, \quad i=1,2,3,
$$

whereas their linear combinations are not

$$
\mathcal{L}_{v_{k}} P_{g} \neq 0, \quad k=1,2 .
$$

Of course, since the vector fields $v_{k}$ pairwise commute, we have

$$
\mathcal{L}_{v_{k}}\left(v_{1} \wedge v_{2} \wedge v_{3}\right)=0, \quad k=1,2,3,
$$

similar to the Hamiltonian mechanics [10, so the three equations $\dot{x}=v_{k}$ can be simultaneously integrated by quadratures.

\subsection{The Turiel type deformations of the canonical Poisson bivector}

Let $\theta, \psi, \phi$ be the Euler nutation, precession, and rotation angles associated with the fixed in space ortonormal frame, respectively. So that the corresponding rotation matrix is

$$
\mathbf{R}=\left(\begin{array}{ccc}
\cos \phi \cos \psi-\cos \theta \sin \psi \sin \phi & \cos \phi \sin \psi+\cos \theta \cos \psi \sin \phi & \sin \phi \sin \theta \\
-\sin \phi \cos \psi-\cos \theta \sin \psi \cos \phi & -\sin \phi \sin \psi+\cos \theta \cos \psi \cos \phi & \cos \phi \sin \theta \\
\sin \theta \sin \psi & -\sin \theta \cos \psi & \cos \theta
\end{array}\right) .
$$


Using the standard definition of angular momenta via variables $p_{\phi}, p_{\theta}$ and $p_{\psi}$

$$
\begin{aligned}
& M_{1}=\left(\frac{\sin \phi}{\sin \theta}\left(\cos \theta p_{\phi}+p_{\psi}\right)-\cos \phi p_{\theta}\right), \\
& M_{2}=\left(\frac{\cos \phi}{\sin \theta}\left(\cos \theta p_{\phi}+p_{\psi}\right)+\sin \phi p_{\theta}\right), \quad M_{3}=-p_{\phi} .
\end{aligned}
$$

we can rewrite the equations of motion (1.1) as equation on six dimensional phase space with coordinates $x=\left(\phi, \theta, \psi, p_{\phi}, p_{\theta}, p_{\psi}\right)$.

If we know solutions $\phi(t), \theta(t), p_{\phi}(t)$ and $p_{\theta}(t)$, we can obtain a precession angle by quadrature

$$
\dot{\psi}=\frac{\omega_{1} \gamma_{1}+\omega_{2} \gamma_{2}}{g\left(\gamma_{1}^{2}+\gamma_{2}^{2}\right)}
$$

see [5]. As usual, momenta associated with the cyclic variable $\psi$ is a constant of motion

$$
p_{\psi}=(\gamma, M)=\text { const }
$$

that allows us to reduce the initial six dimensional phase space $\mathcal{M}$ to four dimensional phase $\hat{\mathcal{M}}$ space with coordinates $\hat{x}=\left(\phi, \theta, p_{\phi}, p_{\theta}\right)$.

This phase space $\hat{\mathcal{M}}$ is diffeomorphic to the cotangent bundle of the two-dimensional unit sphere $T^{*} S^{2}$. According to [17] there are Turiel type deformations of the canonical Poisson bivector

$$
P=\left(\begin{array}{cc}
0 & \mathrm{I} \\
-\mathrm{I} & 0
\end{array}\right)
$$

depending on two arbitrary functions $g(\phi, \theta)$ and $h(\phi, \theta)$

$$
\hat{P}_{g}=\left(\begin{array}{cccc}
0 & 0 & g(\phi, \theta) & 0 \\
* & 0 & 0 & g(\phi, \theta) \\
* & * & 0 & \partial_{\theta} g p_{\phi}-\partial_{\phi} g p_{\theta}-h(\phi, \theta) \\
* & * & * & 0
\end{array}\right) .
$$

Here $\partial_{\phi} g$ and $\partial_{\theta} g$ are derivatives of $g(\phi, \theta)$ by variables $\phi$ and $\theta$, respectively.

In the particular case if $g(\phi, \theta)$ is given by (1.4) and

$$
h(\phi, \theta)=w(\phi, \theta) p_{\psi}, \quad w(\phi, \theta)=g^{-1} d \sin \theta\left(a_{1} \sin ^{2} \phi+a_{2} \cos ^{2} \phi\right),
$$

one gets bivector $\hat{P}_{g}$ (2.3), which was found by Borisov and Mamaev 4. Expression for $g(\phi, \theta)$ is fixed by invariant volume form $\Omega(1.9)$, whereas function $h(\phi, \theta)$ is uniquely defined by the involutivity condition

$$
\left\{H, M^{2}\right\}_{g}=0 \text {. }
$$

Let us consider lifting the Poisson bivector $\hat{P}_{g}$ with arbitrary $g(\phi, \theta)$ and $h(\phi, \theta)=w(\phi, \theta) p_{\psi}$ to the rank-six Poisson bivector on the initial phase space $\mathcal{M}$.

Proposition 3 Two arbitrary functions $g(\phi, \theta)$ and $w(\phi, \theta)$ define the Poisson bivector with linear in momenta entries

$$
\tilde{P}_{g}=\left(\begin{array}{cccccc}
0 & 0 & 0 & g(\phi, \theta) & 0 & 0 \\
* & 0 & 0 & 0 & g(\phi, \theta) & 0 \\
* & * & 0 & u(\phi, \theta) & v(\phi, \theta) & 1 \\
* & * & * & 0 & \partial_{\theta} g p_{\phi}-\partial_{\phi} g p_{\theta}-w(\phi, \theta) p_{\psi} & 0 \\
* & * & * & * & 0 & 0 \\
* & * & * & * & * & 0
\end{array}\right)
$$

on the six dimensional phase space $\mathcal{M}$ with coordinates $x=\left(\phi, \theta, \psi, p_{\phi}, p_{\theta}, p_{\psi}\right)$, if

$$
g\left(\partial_{\phi} v(\phi, \theta)-\partial_{\theta} u(\phi, \theta)\right)+u(\phi, \theta) \partial_{\theta} g-v(\phi, \theta) \partial_{\phi} g-w(\phi, \theta)=0
$$

Bivector $P_{g}$, rank $P_{g}=6$, defines a symplectic form and an invariant volume form with density $g^{-1}(\phi, \theta)$ on the phase space $\mathcal{M}$, which becomes a symplectic manifold. 
The proof is straightforward.

Remark 5 Change of variables

$$
p_{\phi}=g^{-1}\left(p_{\phi}-u(\phi, \theta) p_{\psi}\right), \quad \text { and } \quad p_{\theta}=g^{-1}\left(p_{\theta}-v(\phi, \theta) p_{\psi}\right)
$$

reduces bivector $P_{g}$ to the canonical bivector

$$
P=\left(\begin{array}{cc}
0 & \mathrm{I} \\
-\mathrm{I} & 0
\end{array}\right)
$$

We can say that $P_{g}$ is a deformation of $P$ in the Poisson-Lichnerowicz cohomology [16].

For the Chaplygin ball $g(\phi, \theta)$ and $w(\phi, \theta)$ are given by (1.4) and (2.15), respectively. In this case there is a "simple" partial solution of equation (2.17)

$$
v(\phi, \theta)=0, \quad u(\phi, \theta)=g F(\phi)+\frac{d \cos \theta\left(a_{1} \sin ^{2} \phi+a_{2} \cos ^{2} \phi\right)}{1-d a_{1} \sin ^{2} \phi-d a_{2} \cos ^{2} \phi}
$$

where $F(\phi)$ is an arbitrary function. At $F(\phi)=0$ this particular choice of functions $g, w, v$ and $u$ corresponds to the bivector $P_{g}$ (2.5).

Let us show another solution for the same $g(\phi, \theta)$ and $w(\phi, \theta)$

$$
\begin{aligned}
v(\phi, \theta)= & \frac{g}{\sin \theta \sqrt{1-d a_{1} \sin ^{2} \theta-d a_{3} \cos ^{2} \theta}}\left(E_{1}(\cos \phi, z)+\right. \\
& \left.+\frac{\mathrm{i}\left(1-d a_{3} \cos ^{2} \theta\right) E_{3}\left(z \cos \phi, 1, z^{-1}\right)}{\sin \theta \sqrt{d\left(a_{1}-a_{2}\right)\left(1-d a_{1} \sin ^{2} \theta-d a_{3} \cos ^{2} \theta\right)}}\right), \\
u(\phi, \theta)= & 0 .
\end{aligned}
$$

Here $E_{1}$ and $E_{3}$ are incomplete elliptic integrals of the first and third kind, respectively, and

$$
z=\frac{i \sin \theta \sqrt{d\left(a_{1}-a_{2}\right)}}{\sqrt{1-d a_{1} \sin ^{2} \theta-d a_{3} \cos ^{2} \theta}}, \quad \mathrm{i}=\sqrt{-1} .
$$

Of course, there are a lot of other solutions of the equation (2.17) for the given $g(\phi, \theta)$ and $w(\phi, \theta)$.

Remark 6 We can not put $s_{1}=0$ in the decomposition (2.9) using some special choice of the functions $u(\phi, \theta)$ and $v(\phi, \theta)$.

\subsection{Rank-two Poisson bivectors associated with the Chaplygin ball}

The Lie symmetry analysis provides an algorithm to determine a set of infinitesimal symmetries associated with the given equations of motion. Finding the Jacobi last multiplier and integrals of motion from the Lie symmetries is often a cumbersome procedure. On the other hand it is very easy to get rank-two Poisson structures directly from symmetries [12].

Namely, for the Chaplygin ball we have two families of pairwise commuting vector fields

$$
u_{1}=P_{g} \mathrm{~d} f_{1}, \quad u_{2}=P_{g} \mathrm{~d} f_{2}, \quad u_{3}=P_{g} \mathrm{~d} f_{3}, \quad\left[u_{i}, u_{j}\right]=0
$$

and

$$
v_{1}=u_{1}-s_{1} u_{3}, \quad v_{2}=u_{2}-s_{2} u_{3}, \quad v_{3}=u_{3}, \quad\left[v_{i}, v_{j}\right]=0 .
$$

Using these vector fields we can construct two families of bivectors

$$
P_{u}^{(i . j)}=u_{i} \wedge u_{j}, \quad \text { and } \quad P_{v}^{(i . j)}=v_{i} \wedge v_{j}, \quad i, j=1,2,3 .
$$


It is easy to prove that the Schouten bracket for these bivectors are equal to zero, for instance

$$
\left[P_{v}^{(i, j)}, P_{v}^{(i, j)}\right]=2 v_{i} \wedge v_{j} \wedge\left[v_{i}, v_{j}\right]=0
$$

It means that we have two families of the Poisson bivectors having a constant rank two, see [12. Entries of these bivectors are the fourth order polynomials in momenta whereas entries of initial Poisson bivector $P_{g}$ are linear polynomials.

Associated with the Poisson bivector $P_{v}^{(i, j)}$ brackets is equal to

$$
\{F, G\}_{v}^{(i, j)}=\left(\mathrm{d} F \wedge \mathrm{d} G, P_{v}^{(i, j)}\right)=\mathcal{L}_{v_{i}} F \cdot \mathcal{L}_{v_{j}} G-\mathcal{L}_{v_{i}} G \cdot \mathcal{L}_{v_{j}} F
$$

Sequently, integrals of motion $f_{1}, f_{2}$ and $f_{3}$ are the Casimir functions for these Poisson brackets because

$$
\mathcal{L}_{v_{k}} f_{m}=0, \quad \forall k, m=1,2,3 .
$$

In fact we have two families of compatible Poisson bivectors, i.e. linear combinations

$$
P_{u}=\lambda P_{u}^{(1,2)}+\mu P_{u}^{(1,3)}+\nu P_{u}^{(2,3)}, \quad \text { and } \quad P_{v}=\lambda P_{v}^{(1,2)}+\mu P_{v}^{(1,3)}+\nu P_{v}^{(2,3)}
$$

are also the Poisson bivectors for any $\lambda, \mu$ and $\nu$.

In [3] we used linear combinations of the rank-two Poisson bivectors in order to get rankfour Poisson bivectors for the nonholonomic Routh sphere. It will be interesting to study similar linear combinations in the Chaplygin case too.

\section{Possible generalisations}

Using functionally independent integrals of motion in involution we construct a set of commuting Hamiltonian vector fields

$$
u_{k}=P \mathrm{~d} f_{k}, \quad\left[u_{i}, u_{j}\right]=0,
$$

which form a basis with a necessary number of generators for applicability of the Lie integrability theorem. Other combinations could be taken

$$
v_{i}=\sum s_{i k} u_{k}
$$

in order to include the original vector field $v_{1}=g X$ and the necessary number of symmetries into the abelian (solvable) algebra. Coefficient $s_{i k}$ may be obtained by direct integration of a system of equations similar to (2.11).

Remark 7 Recall that in the toric integrability theory we also consider special linear combinations of the initial commuting Hamiltonian vector fields in order to get the so-called fundamental vector fields of the torus action [13, 10].

It is easy to see that in this direct method we do not use the known volume form $\Omega(1.9)$, which is a key element of the Euler-Jacobi integrability theorem. So, we hope that there is another constructive method of finding vector fields $v_{k}$ (3.1) using the volume form, its relation with symmetries (1.13), torus action theory, modular vector fields or something else.

\subsection{Motion in the Brun potential field}

Let us consider the motion of the Chaplygin ball in the so-called Brun potential field [8]. In order to get the corresponding vector field $X_{b}$ we have to add a momenta of external force to the equation for the angular momenta in (1.1)

$$
M=M \times \omega+\frac{\partial U}{\partial \gamma} \times \gamma, \quad U=c\left(a_{2}, a_{3} \gamma_{1}^{2}+a_{1} a_{3} \gamma_{2}^{2}+a_{1} a_{2} \gamma_{3}^{2}\right)
$$


In this case we have nine integrals of motion $C_{1}, \ldots, C_{6}$ and

$$
f_{1}=\frac{1}{2}(M, \omega)+U, \quad f_{2}=M^{2}-2 c\left(a_{1} \gamma_{1}^{2}+a_{2} \gamma_{2}^{2}+a_{3} \gamma_{3}^{2}\right), \quad f_{3}=(\gamma, M)
$$

and the same invariant volume form (1.9). However, functions $J_{1}=(\alpha, M)$ and $J_{2}=(\beta, M)$ are no longer constants of motion and we cannot apply the Euler-Jacobi integrability theorem in this case.

Of course, equations for $\gamma$ and $M$ on the six dimensional phase space $\hat{\mathcal{M}}$ are integrable by quadratures according to the Euler-Jacobi theorem, and, after integration of these equations of motion for $\gamma$ and $M$, we can integrate equations for the remaining variables $\alpha$ and $\beta$ by quadratures as well. But we do not have a formal proof of integrability on 12-th dimensional phase space $\mathcal{M}$.

According to [16], vector filed $\hat{X}_{b}$ on the six-dimensional phase space $\hat{\mathcal{M}}$ is a conformally Hamiltonian vector field

$$
\hat{X}_{b}=g^{-1} \hat{P}_{g} \mathrm{~d}(H+U)
$$

where $\hat{P}_{g}$ is the same Poisson bivector (2.3) as for the free Chapligin ball.

The lifting of the Poison bivector $\hat{P}_{g}$ on the 12-dimensional phase space is given by (2.5). Using this Poisson bivector and integrals of motion we can construct three independent commuting vector fields

$$
u_{2}=P_{g} \mathrm{~d} f_{1}, \quad u_{3}=P_{g} \mathrm{~d} f_{2}, \quad u_{4}=P_{g} \mathrm{~d} f_{3}, \quad\left[u_{i}, u_{j}\right]=0 .
$$

The original vector field $X_{b}(1.13 .2)$ is a linear combination

$$
X=g^{-1}\left(u_{2}-s_{1} u_{4}\right),
$$

of these Hamiltonian vector fields, where $s_{1}$ is the same coefficients as in (2.9).

As in the previous Section we can apply the Chaplygin change of time and define the three independent commuting vector fields

$$
v_{1}=g X_{b}=u_{2}-s_{1} u_{4}, \quad v_{2}=u_{3}-\left(s_{2}+\delta s\right) u_{4}, \quad v_{3}=u_{4} .
$$

Here $s_{2}$ is given by (2.12) and $\delta s$ is the solution of the following differential equation

$$
\left\{f_{1}, \delta s\right\}=4 c d\left(a_{1}-a_{2}\right)\left(a_{2}-a_{3}\right)\left(a_{3}-a_{1}\right) \gamma_{1} \gamma_{2} \gamma_{3}
$$

where $f_{1}$ is mechanical energy. We do not have an explicit observable solution of this equation.

\subsection{Motion of the contact point}

The trajectory of the contact point on the plane is also important for understanding the ball's motion in absolute space. The equations of motion of the contact point can be obtained using the condition that its velocity is equal to zero in the body frame

$$
v=r \times \omega=0 .
$$

Here $v$ is the velocity of the center of mass, $r=b \gamma$ is a vector, connecting the center of mass with the contact point. In terms of projections on the axes of coordinates this condition looks like

$$
\dot{x}=(v, \alpha)=b(\omega, \beta), \quad \dot{y}=(v, \beta)=-b(\omega, \alpha) .
$$

Here $x$ and $y$ are coordinates of the contact point on the plane and $b$ is the ball's radius [5, 7].

Thus, on the phase space $\mathcal{M}_{c}, \operatorname{dim} \mathcal{M}_{c}=14$ we have a vector field $X_{c}$ defined by equations of motion (1.1) and (3.6). As above, we can try to prove the integrability of this vector field using the Poisson geometry methods. 
Proposition 4 By adding Poisson brackets between new variables

$$
\{x, y\}_{g}=1
$$

to the Poisson brackets $\{.,\}_{g}$ (2.5) one gets a rank eight Poisson bivector $P_{c}$ on the 14-th dimensional phase space with the Casimir functions $C_{1}, \ldots, C_{6}$.

In this case the 14-th dimensional vector field $X_{c}$ is a linear combination of the Hamiltonian vector fields

$$
\left.X_{c}=g^{-1}\left(P_{c} \mathrm{~d} f_{1}-s_{1} P_{c} \mathrm{~d} f_{3}-s_{2} P_{c} \mathrm{~d} x-s_{3} P_{c} \mathrm{~d} y\right)\right),
$$

which has two symmetries associated with the cyclic variables $x$ and $y$. Here

$$
s_{2}=b g(\omega, \beta) \quad \text { and } \quad s_{3}=b g(\omega, \alpha) .
$$

So, we have nine integrals of motion in involution $C_{1}, \ldots, C_{6}, f_{1} \ldots, f_{3}$ and five commuting vector fields

$$
u_{1}=P_{c} \mathrm{~d} f_{1}, \quad u_{2}=P_{c} \mathrm{~d} f_{2}, \quad u_{3}=P_{c} \mathrm{~d} f_{3}, \quad u_{4}=P_{c} \mathrm{~d} x, \quad u_{5}=P_{c} \mathrm{~d} y .
$$

After the change of time the initial vector field $v_{1}=g X_{c}$ commutes with only two symmetry fields

$$
\left[v_{1}, u_{4}\right]=\left[v_{1}, u_{5}\right]=0 \quad \text { and } \quad\left[v_{1}, u_{k}\right] \neq 0, \quad k=1,2,3 .
$$

Therefore, we have to add two linear combinations of $u_{k}$

$$
v_{k}=\sum_{j=1}^{5} s_{k j} u_{j}, \quad k=2,3 .
$$

to the field $v_{1}$ and its symmetries $v_{4}=u_{4}, v_{5}=u_{5}$ associated with cyclic variables $x, y$. Here $s_{k j}$ are some locally defined functions on phase space.

Supposing that $v_{1}, \ldots, v_{5}$ generate a solvable (nilpotent or Abelian) algebra one gets a system of differential equations on $s_{k j}$ (3.8) similar to (2.11) and (3.5), which we have to integrate.

In some sense we are looking for an inversion of the Routh procedure, which in its original form [15], was concerned with eliminating the generalized velocities corresponding to ignorable or cyclic coordinates from a Lagrangian problem. The modern discussion of the Routh procedure and its inversion based on the notion of anholonomic frames may be found in 6].

We would like to thank Alexey Borisov and Ivan Mamaev for genuine interest and helpful discussions. This work was partially supported by RFBR grant 13-01-00061.

\section{References}

[1] V. I. Arnold, V.V. Kozlov, A. I. Neîshtadt, Mathematical Aspects of Classical and Celestial Mechanics, Encyclopaedia Math. Sci., vol. 3, Berlin: Springer, 1993, pp. 1-29.

[2] L. Bianchi, Lezione sulla teoria dei gruppi continui finiti di transformazioni, Enrico Spoerri, Pisa, 1918.

[3] I. A. Bizayev, A. V. Tsiganov, On the Routh sphere problem, J. Phys. A: Math. Theor., v. 46, 085202 (11pp), 2013.

[4] A.V. Borisov, I.S. Mamaev, The Chaplygin problem of the rolling motion of a ball is Hamiltonian, Math. Notes, v. 70, n. 5, p. 720-723, 2001.

[5] S.A. Chaplygin, On a Ball's Rolling on a Horizontal Plane, Mathematical collection of the Moscow Mathematical Society, v.24, p.139-168, 1903. 
[6] M. Crampin, T. Mestdag, Routh's procedure for non-Abelian symmetry groups, J. Math. Phys., v. 49, 032901, 2008.

[7] A.A. Kilin, The dynamics of Chaplygin ball: the qualitative and computer analysis, Regular and Chaotic Dynamics, v. 6, pp. 291306, 2001.

[8] V.V. Kozlov, On the integration theory of equations of nonholonomic mechanics, Adv. in Mech., v.8, no.3, pp. 85-107, 1985.

[9] V.V. Kozlov, The Euler-Jacobi-Lie integrability theorem, Regul. Chaotic Dyn., 18 (4), pp. 329-343, 2013.

[10] C. Laurent-Gengoux, E. Miranda, P. Vanhaecke, Action-angle coordinates for integrable systems on Poisson manifolds, Int. Math. Res. Notices, v.8, pp. 1839-1869, 2011.

[11] S. Lie, Vorlesungen über Differentialgleichungen mit bekannten infinitesimalen Transformationen, B.G. Teubner edition, Leipzig, 1912.

[12] J.E. Marsden and T.S. Ratiu, Introduction to Mechanics and Symmetry, 2nd Edi- tion, Texts in Applied Mathematics, vol. 17, Springer, Berlin, 1999.

[13] N. N. Nekhoroshev, Types of integrability on a submanifold and generalizations of Gordon's theorem, Trans. Moscow Math. Soc., v.66, pp.169241, 2005.

[14] P.J. Olver, Applications of Lie groups to differential equations, Springer; 2-nd edition, 513 p., 2000.

[15] E.J. Routh, Advanced Rigid Bodies Dynamics, MacMillan and Co., London 1884, Reprint: Advanced Dynamics of a System of Rigid Bodies, Dover Publications, New York, 1960.

[16] A. V. Tsiganov, Integrable Euler top and nonholonomic Chaplygin ball, Journal of Geometric Mechanics, v.3, n.3, p.337 - 362, 2011.

[17] A. V. Tsiganov, One family of conformally Hamiltonian systems, Theor. Math. Phys., v.173(2), pp.1481-1497, 2012.

[18] A. V. Tsiganov, On the Poisson structures for the nonholonomic Chaplygin and Veselova problems, Reg. Chaotic Dyn., v.17, n.5, p. 439 - 450, 2012.

[19] A. V. Tsiganov, On generalized nonholonomic Chaplygin sphere problem, Int. J. Geom. Meth. in Mod. Phys., v.10, 1320008, (8 pages), 2013.

[20] A. V. Tsiganov, On the nonholonomic Stübler model, Nonlinear Dynamics \& Mobile Robotics, v.1, p.87-97, 2013. 